\title{
CODATA RECOMMENDED VALUES OF THE FUNDAMENTAL PHYSICAL CONSTANTS: 2014
}

NIST SP 961 (Sept/2015) Values from: P. J. Mohr, D. B. Newell, and B. N. Taylor, arXiv:1507.07956

A more extensive listing of constants is available in the above reference and on the NIST Physics Laboratory Web site physics.nist.gov/constants.

The number in parentheses is the one-standard-deviation uncertainty in the last two digits of the given value.

\begin{tabular}{|c|c|c|c|c|c|c|c|}
\hline Quantity & Symbol & Numerical value & Unit & Quantity & Symbol & Numerical value & Unit \\
\hline speed of light in vacuum & $c, c_{0}$ & 299792458 (exact) & $\mathrm{m} \mathrm{s}^{-1}$ & muon $g$-factor $-2\left(1+a_{\mu}\right)$ & $g_{\mu}$ & $-2.0023318418(13)$ & \\
\hline magnetic constant & $\mu_{0}$ & $4 \pi \times 10^{-7}$ (exact) & $\mathrm{N} \mathrm{A}^{-2}$ & muon-proton magnetic moment ratio & $\mu_{\mu} / \mu_{\mathrm{p}}$ & $-3.183345142(71)$ & \\
\hline & & $=12.566370614 \ldots \times 10^{-7}$ & $\mathrm{~N} \mathrm{~A}^{-2}$ & proton mass & & $1.672621898(21) \times 10^{-27}$ & $\mathrm{~kg}$ \\
\hline electric constant $1 / \mu_{0} c^{2}$ & $\epsilon_{0}$ & $8.854187817 \ldots \times 10^{-12}$ & $\mathrm{~F} \mathrm{~m}^{-1}$ & in $u$ & & $1.007276466879(91)$ & $\mathrm{u}$ \\
\hline Newtonian constant of gravitation & $G$ & $6.67408(31) \times 10^{-11}$ & $\mathrm{~m}^{3} \mathrm{~kg}^{-1} \mathrm{~s}^{-2}$ & energy equivalent in $\mathrm{MeV}$ & $m_{\mathrm{p}} c^{2}$ & $938.2720813(58)$ & $\mathrm{MeV}$ \\
\hline $\begin{array}{c}\text { Planck constant } \\
\text { in } \mathrm{eV} \mathrm{s}\end{array}$ & $h$ & $\begin{array}{l}6.626070040(81) \times 10^{-34} \\
4.135667662(25) \times 10^{-15}\end{array}$ & $\begin{array}{l}\mathrm{J} \mathrm{s} \\
\mathrm{eV} \mathrm{s}\end{array}$ & $\begin{array}{l}\text { proton-electron mass ratio } \\
\text { proton magnetic moment }\end{array}$ & $\begin{array}{l}m_{\mathrm{p}} / m_{\mathrm{e}} \\
\mu_{\mathrm{p}}\end{array}$ & $\begin{array}{l}1836.15267389(17) \\
1.4106067873(97) \times 10^{-26}\end{array}$ & $\mathrm{~J} \mathrm{~T}^{-1}$ \\
\hline$h / 2 \pi$ & $\hbar$ & $1.054571800(13) \times 10^{-34}$ & $\mathrm{~J} \mathrm{~s}$ & to nuclear magneton ratio & $\begin{array}{l}\mu_{\mathrm{p}} \\
\mu_{\mathrm{p}} / \mu_{\mathrm{N}}\end{array}$ & $2.7928473508(85)$ & \\
\hline in $\mathrm{eV} \mathrm{s}$ & & $6.582119514(40) \times 10^{-16}$ & $\mathrm{eVs}$ & proton magnetic shielding correction $1-\mu_{\mathrm{p}}^{\prime} / \mu_{\mathrm{p}}$ & $\sigma_{\mathrm{p}}^{\prime}$ & $25.691(11) \times 10^{-6}$ & \\
\hline elementary charge & $e$ & $1.6021766208(98) \times 10^{-19}$ & $\mathrm{C}$ & $\left(\mathrm{H}_{2} \mathrm{O}\right.$, sphere, $\left.25{ }^{\circ} \mathrm{C}\right)$ & & & \\
\hline magnetic flux quantum $h / 2 e$ & $\Phi_{0}$ & $2.067833831(13) \times 10^{-15}$ & $\mathrm{~Wb}$ & proton gyromagnetic ratio $2 \mu_{\mathrm{p}} / \hbar$ & $\gamma_{\mathrm{p}}$ & $2.675221900(18) \times 10^{8}$ & $\mathrm{~s}^{-1} \mathrm{~T}^{-1}$ \\
\hline Josephson constant $2 e / h$ & $K_{\mathrm{J}}$ & $483597.8525(30) \times 10^{9}$ & $\mathrm{~Hz} \mathrm{~V}^{-1}$ & & $\gamma_{\mathrm{p}} / 2 \pi$ & $42.57747892(29)$ & $\mathrm{MHz} \mathrm{T}^{-1}$ \\
\hline von Klitzing constant $h / e^{2}=\mu_{0} c / 2 \alpha$ & $R_{\mathrm{K}}$ & $25812.8074555(59)$ & & shielded proton gyromagnetic ratio $2 \mu_{\mathrm{p}}^{\prime} / \hbar$ & $\gamma_{\mathrm{p}}^{\prime}$ & $2.675153171(33) \times 10^{8}$ & $\mathrm{~s}^{-1} \mathrm{~T}^{-1}$ \\
\hline $\begin{array}{l}\text { Bohr magneton e } e / 2 m_{\mathrm{e}} \\
\quad \text { in } \mathrm{eV} \mathrm{T}^{-1}\end{array}$ & $\mu_{\mathrm{B}}$ & $\begin{array}{l}927.4009994(57) \times 10^{-26} \\
5.7883818012(26) \times 10^{-5}\end{array}$ & $\begin{array}{l}\mathrm{J} \mathrm{T}^{-1} \\
\mathrm{eV} \mathrm{T}^{-1}\end{array}$ & $\left(\mathrm{H}_{2} \mathrm{O}\right.$, sphere, $\left.25^{\circ} \mathrm{C}\right)$ & $\gamma_{p}^{\prime} / 2 \pi$ & $42.57638507(53)$ & $\mathrm{MHz} \mathrm{T}^{-1}$ \\
\hline $\begin{array}{l}\text { nuclear magneton } e \hbar / 2 m_{\mathrm{p}} \\
\quad \text { in } \mathrm{eV} \mathrm{T}^{-1}\end{array}$ & $\mu_{\mathrm{N}}$ & $\begin{array}{l}5.050783699(31) \times 10^{-27} \\
3.1524512550(15) \times 10^{-8}\end{array}$ & $\begin{array}{l}\mathrm{J} \mathrm{T}^{-1} \\
\mathrm{eV} \mathrm{T}^{-1}\end{array}$ & $\begin{array}{l}\text { neutron mass in } \mathrm{u} \\
\quad \text { energy equivalent in } \mathrm{MeV}\end{array}$ & $\begin{array}{l}m_{\mathrm{n}} \\
m_{\mathrm{n}} c^{2}\end{array}$ & $\begin{array}{l}1.00866491588(49) \\
939.5654133(58)\end{array}$ & $\begin{array}{l}\mathrm{u} \\
\mathrm{MeV}\end{array}$ \\
\hline fine-structure constant $e^{2} / 4 \pi \epsilon_{0} \hbar c$ & $\alpha$ & $7.2973525664(17) \times 10^{-3}$ & & neutron-proton mass ratio & $m_{\mathrm{n}} / m_{\mathrm{p}}$ & $1.00137841898(51)$ & \\
\hline inverse fine-structure constant & $\alpha^{-1}$ & $137.035999139(31)$ & & neutron magnetic moment & & $-0.96623650(23) \times 10^{-26}$ & $\mathrm{~J} \mathrm{~T}^{-1}$ \\
\hline Rydberg constant $\alpha^{2} m_{\mathrm{e}} c / 2 h$ & $R_{\infty}$ & $10973731.568508(65)$ & $\mathrm{m}^{-1}$ & to nuclear magneton ratio & $\mu_{\mathrm{n}} / \mu_{\mathrm{N}}$ & $-1.91304273(45)$ & \\
\hline & $R_{\infty} c$ & $3.289841960355(19) \times 10^{15}$ & $\mathrm{~Hz}$ & deuteron mass in $u$ & $m_{\mathrm{d}}$ & $2.013553212745(40)$ & $\mathrm{u}$ \\
\hline energy equivalent in $\mathrm{eV}$ & $R_{\infty} h c$ & $13.605693009(84)$ & $\mathrm{eV}$ & energy equivalent in $\mathrm{MeV}$ & $m_{\mathrm{d}} c^{2}$ & $1875.612928(12)$ & $\mathrm{MeV}$ \\
\hline Bohr radius $\alpha / 4 \pi R_{\infty}=4 \pi \epsilon_{0} \hbar^{2} / m_{\mathrm{e}} e^{2}$ & $a_{0}$ & $0.52917721067(12) \times 10^{-10}$ & $\mathrm{~m}$ & deuteron-proton mass ratio & $m_{\mathrm{d}} / m_{\mathrm{p}}$ & $1.99900750087(19)$ & \\
\hline $\begin{array}{l}\text { Hartree energy } e^{2} / 4 \pi \epsilon_{0} a_{0}=2 R_{\infty} h c=\alpha^{2} m_{\mathrm{e}} c^{2} \\
\quad \text { in eV }\end{array}$ & $E_{\mathrm{h}}$ & $\begin{array}{l}4.359744650(54) \times 10^{-18} \\
27.21138602(17)\end{array}$ & $\begin{array}{l}\mathrm{J} \\
\mathrm{eV}\end{array}$ & $\begin{array}{l}\text { deuteron magnetic moment } \\
\text { to nuclear magneton ratio }\end{array}$ & $\begin{array}{l}\mu_{\mathrm{d}} \\
\mu_{\mathrm{d}} / \mu_{\mathrm{N}}\end{array}$ & $\begin{array}{l}0.4330735040(36) \times 10^{-26} \\
0.8574382311(48)\end{array}$ & $\mathrm{J} \mathrm{T}^{-1}$ \\
\hline $\begin{array}{c}\text { electron mass } \\
\text { in } u\end{array}$ & $m_{\mathrm{e}}$ & $\begin{array}{l}9.10938356(11) \times 10^{-31} \\
5.48579909070(16) \times 10^{-4}\end{array}$ & $\begin{array}{l}\mathrm{kg} \\
\mathrm{u}\end{array}$ & $\begin{array}{l}\text { helion }\left({ }^{3} \mathrm{He} \text { nucleus }\right) \text { mass in u } \\
\text { energy equivalent in } \mathrm{MeV}\end{array}$ & $\begin{array}{l}m_{\mathrm{h}} \\
m_{\mathrm{h}} c^{2}\end{array}$ & $\begin{array}{l}3.01493224673(12) \\
2808.391586(17)\end{array}$ & $\begin{array}{l}\mathrm{u} \\
\mathrm{MeV}\end{array}$ \\
\hline energy equivalent in $\mathrm{MeV}$ & $m_{\mathrm{e}} c^{2}$ & $0.5109989461(31)$ & $\mathrm{MeV}$ & shielded helion magnetic moment & $\mu_{\mathrm{h}}^{\prime}$ & $-1.074553080(14) \times 10^{-26}$ & $\mathrm{~J} \mathrm{~T}^{-1}$ \\
\hline electron-muon mass ratio & $m_{\mathrm{e}} / m_{\mu}$ & $4.83633170(11) \times 10^{-3}$ & & (gas, sphere, $25^{\circ} \mathrm{C}$ ) & & & \\
\hline electron-proton mass ratio & $m_{\mathrm{e}} / m_{\mathrm{p}}$ & $5.44617021352(52) \times 10^{-4}$ & & to Bohr magneton ratio & $\mu_{\mathrm{h}}^{\prime} / \mu_{\mathrm{B}}$ & $-1.158671471(14) \times 10^{-3}$ & \\
\hline electron charge to mass quotient & $-e / m_{\mathrm{e}}$ & $-1.758820024(11) \times 10^{11}$ & $\mathrm{C} \mathrm{kg}^{-1}$ & to nuclear magneton ratio & $\mu_{\mathrm{h}}^{\prime} / \mu_{\mathrm{N}}$ & -2.127 & \\
\hline Compton wavelength $h / m_{\mathrm{e}} c$ & $\lambda_{\mathrm{C}}$ & $2.4263102367(11) \times 10^{-12}$ & $\mathrm{~m}$ & alpha particle mass in $\mathrm{u}$ & $m_{\alpha}$ & $4.001506179127(63)$ & $\mathrm{u}$ \\
\hline$\lambda_{\mathrm{C}} / 2 \pi=\alpha a_{0}=\alpha^{2} / 4 \pi R_{\infty}$ & $\hbar_{\mathrm{C}}$ & $386.15926764(18) \times 10^{-15}$ & $\mathrm{~m}$ & energy equivalent in $\mathrm{MeV}$ & $m_{\alpha} c^{2}$ & 3727.3 & $\mathrm{MeV}$ \\
\hline classical electron radius $\alpha^{2} a_{0}$ & $r_{\mathrm{e}}$ & $2.8179403227(19) \times 10^{-15}$ & & Avogadro constant & $N_{\mathrm{A}}, L$ & $6.022140857(74) \times 10^{23}$ & $\mathrm{~mol}^{-1}$ \\
\hline Thomson cross section $(8 \pi / 3) r_{\mathrm{e}}^{2}$ & $\sigma_{\mathrm{e}}$ & $0.66524587158(91) \times 10^{-28}$ & & atomic mass constant $\frac{1}{12} m\left({ }^{12} \mathrm{C}\right)=1 \mathrm{u}$ & $m_{\mathrm{u}}$ & $1.660539040(20) \times 10^{-27}$ & $\mathrm{~kg}$ \\
\hline electron magnetic moment & & $-928.4764620(57) \times 10^{-26}$ & $\mathrm{~J} \mathrm{~T}^{-1}$ & energy equivalent in $\mathrm{MeV}$ & $m_{\mathrm{u}} c^{2}$ & $931.4940954(57)$ & $\mathrm{MeV}$ \\
\hline to Bohr magneton ratio & $\mu_{\mathrm{e}} / \mu_{\mathrm{B}}$ & $-1.00115965218091(26)$ & & Faraday constant $N_{\mathrm{A}} e$ & $F$ & $96485.33289(59)$ & $\mathrm{C} \mathrm{mol}{ }^{-1}$ \\
\hline to nuclear magneton ratio & $\mu_{\mathrm{e}} / \mu_{\mathrm{N}}$ & -1838.2819723 & & mol & $R$ & 8.314 & $\mathrm{~J} \mathrm{~mol}^{-1} \mathrm{~K}^{-1}$ \\
\hline electron magnetic moment anomaly $\left|\mu_{\mathrm{e}}\right| / \mu_{\mathrm{B}}-1$ & $a_{\mathrm{e}}$ & $1.15965218091(26) \times 10^{-3}$ & & Boltzmann constant $R / N_{\mathrm{A}}$ & $k$ & $1.38064852(79) \times 10^{-23}$ & $\mathrm{~J} \mathrm{~K}^{-1}$ \\
\hline electron $g$-factor $-2\left(1+a_{\mathrm{e}}\right)$ & & $-2.00231930436182(52)$ & & in $V K^{-1}$ & & $8.6173303(50) \times 10^{-5}$ & $\mathrm{eV} \mathrm{K}^{-1}$ \\
\hline $\begin{array}{l}\text { electron-proton magnetic moment ratio } \\
\text { muon mass in u }\end{array}$ & $\begin{array}{l}\mu_{\mathrm{e}} / \mu_{\mathrm{p}} \\
m_{\mu}\end{array}$ & $\begin{array}{l}-658.2106866(20) \\
0.1134289257(25)\end{array}$ & $\mathrm{u}$ & $\begin{array}{l}\text { molar volume of ideal gas } R T / p \\
(T=273.15 \mathrm{~K}, p=101.325 \mathrm{kPa})\end{array}$ & $V_{\mathrm{m}}$ & $22.413962(13) \times 10^{-}$ & $\mathrm{m}^{3}$ \\
\hline energy equivalent in $\mathrm{MeV}$ & $m_{\mu} c^{2}$ & $105.6583745(24)$ & $\mathrm{MeV}$ & Stefan-Boltzmann constant $\pi^{2} k^{4} / 60 \hbar^{3} c^{2}$ & $\sigma$ & $5.670367(13) \times 10^{-8}$ & $\mathrm{~W} \mathrm{~m}^{-2} \mathrm{~K}^{-4}$ \\
\hline muon-electron mass ratio & $m_{\mu} / m_{\mathrm{e}}$ & $206.7682826(46)$ & & first radiation constant $2 \pi h c^{2}$ & $c_{1}$ & $3.741771790(46) \times 10^{-16}$ & $\mathrm{~W} \mathrm{~m}^{2}$ \\
\hline muon magnetic moment & & $-4.49044826(10) \times 10^{-26}$ & $\mathrm{~J} \mathrm{~T}^{-1}$ & second radiation constant $h c / k$ & $c_{2}$ & $1.43877736(83) \times 10^{-2}$ & $\mathrm{~m} \mathrm{~K}$ \\
\hline to Bohr magneton ratio & $\mu_{\mu} / \mu_{\mathrm{B}}$ & $-4.84197048(11) \times 10^{-3}$ & & Wien displacement law constant & & & \\
\hline to nuclear magneton ratio & $\mu_{\mu} / \mu_{\mathrm{N}}$ & $-8.89059705(20)$ & & $b=\lambda_{\max } T=c_{2} / 4.965114231 \ldots$ & & $2.8977729(17) \times 10^{-3}$ & $\mathrm{~m} \mathrm{~K}$ \\
\hline muon magnetic moment anomaly & & & & $\mathrm{Cu}$ x unit: $\lambda\left(\mathrm{Cu} \mathrm{K} \alpha_{1}\right) / 1537.400$ & $\mathrm{xu}\left(\mathrm{Cu} K \alpha_{1}\right)$ & $1.00207697(28) \times 10^{-13}$ & $\mathrm{~m}$ \\
\hline$\left|\mu_{\mu}\right| /\left(e \hbar / 2 m_{\mu}\right)-1$ & $a_{\mu}$ & $1.16592089(63) \times 10^{-3}$ & & Mo $\mathrm{x}$ unit: $\lambda\left(\mathrm{Mo} \mathrm{K \alpha}_{1}\right) / 707.831$ & $\mathrm{xu}\left(\mathrm{Mo} \mathrm{K} \alpha_{1}\right)$ & $1.00209952(53) \times 10^{-13}$ & $\mathrm{~m}$ \\
\hline \multicolumn{8}{|c|}{ Energy equivalents } \\
\hline $\begin{array}{ll}\left(1 \mathrm{~m}^{-1}\right) c & =299792458 \mathrm{~Hz} \\
\left(1 \mathrm{~m}^{-1}\right) h c / k & =1.43877736(83) \times 10^{-2} \mathrm{~K} \\
\left(1 \mathrm{~m}^{-1}\right) h c & =1.2398419739(76) \times 10^{-6} \mathrm{eV} \\
\left(1 \mathrm{~m}^{-1}\right) h / c & =1.33102504900(61) \times 10^{-15} \mathrm{u} \\
(1 \mathrm{~Hz}) / c & =3.335640951 \ldots \times 10^{-9} \mathrm{~m}^{-1}\end{array}$ & \multicolumn{3}{|c|}{$\begin{array}{l}(1 \mathrm{~Hz}) h / k=4.7992447(28) \times 10^{-11} \mathrm{~K} \\
(1 \mathrm{~Hz}) h=4.135667662(25) \times 10^{-15} \mathrm{eV} \\
(1 \mathrm{~K}) k / h c=69.503457(40) \mathrm{m}^{-1} \\
(1 \mathrm{~K}) k / h=2.0836612(12) \times 10^{10} \mathrm{~Hz} \\
(1 \mathrm{~K}) k=8.6173303(50) \times 10^{-5} \mathrm{eV}\end{array}$} & $\begin{array}{l}(1 \mathrm{~J}) \quad=6.241509126(38) \times 10^{18} \mathrm{eV} \\
(1 \mathrm{eV})=1.6021766208(98) \times 10^{-19} \mathrm{~J} \\
(1 \mathrm{eV}) / h c=8.065544005(50) \times 10^{5} \mathrm{~m}^{-1} \\
(1 \mathrm{eV}) / h=2.417989262(15) \times 10^{14} \mathrm{~Hz} \\
(1 \mathrm{eV}) / k=1.16045221(67) \times 10^{4} \mathrm{~K}\end{array}$ & \multicolumn{3}{|c|}{ 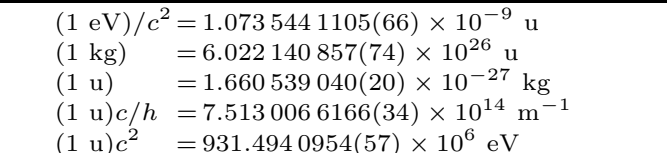 } \\
\hline
\end{tabular}

NI

National Institute of Standards and Technology • U.S. Department of Commerce 